\title{
Assessment of Progesterone Receptors in Breast Carcinoma by PET with $21-{ }^{18}$ F-Fluoro-16 $\alpha, 17 \alpha-\left[(R)-\left(1^{\prime}\right.\right.$ - $\alpha$-furylmethylidene) Dioxy]-19-Norpregn-4-Ene-3,20-Dione
}

\author{
Farrokh Dehdashti ${ }^{1-3}$, Richard Laforest ${ }^{1-3}$, Feng Gao ${ }^{2-4}$, Rebecca L. Aft ${ }^{2,3,5}$, Carmen S. Dence ${ }^{1,3}$, Dong Zhou ${ }^{1,3}$, \\ Kooresh I. Shoghi ${ }^{1-3}$, Barry A. Siegel ${ }^{1-3}$, John A. Katzenellenbogen 6 , and Michael J. Welch ${ }^{1-3}$ \\ ${ }^{1}$ Divisions of Nuclear Medicine and Radiological Sciences, Edward Mallinckrodt Institute of Radiology, St. Louis, Missouri; \\ ${ }^{2}$ Alvin J. Siteman Cancer Center, St. Louis, Missouri, ${ }^{3}$ Washington University School of Medicine, St. Louis, Missouri; \\ ${ }^{4}$ Division of Biostatistics, University of Illinois, Urbana, Illinois; ${ }^{5}$ Department of Surgery, University of Illinois, Urbana, \\ Illinois; and ${ }^{6}$ Department of Chemistry, University of Illinois, Urbana, Illinois
}

This first-in-human study was designed to evaluate the safety and dosimetry of the progesterone analog $21-{ }^{18} \mathrm{~F}$-fluoro- $16 \alpha, 17 \alpha-[(R)-$ ( $1^{\prime}$ - $\alpha$-furylmethylidene)dioxy]-19-norpregn-4-ene-3,20-dione $\left({ }^{18} \mathrm{~F}-\right.$ FFNP), as well the feasibility of imaging tumor progesterone receptors (PRs) by PET in breast cancer. Methods: Women with breast cancer underwent PET with ${ }^{18} \mathrm{~F}-\mathrm{FFNP}$. Tumor ${ }^{18} \mathrm{~F}-\mathrm{FFNP}$ uptake was assessed semiquantitatively by determining maximum standardized uptake value and tumor-to-normal breast $(\mathrm{T} / \mathrm{N})$ activity ratio and by Logan graphical analysis. The PET results were correlated with estrogen receptor (ER) and PR status, assessed by in vitro assays of the tumor tissue. The biodistribution of ${ }^{18} \mathrm{~F}-\mathrm{FFNP}$ was measured in patients by whole-body PET, and human dosimetry was estimated. Results: Twenty patients with 22 primary breast cancers (16 PR-positive [PR+] and 6 PR-negative [PR-]) were evaluated. Tumor maximum standardized uptake value was not significantly different in PR + and PR- cancers (mean $\pm S D$, $2.5 \pm 0.9$ vs. $2.0 \pm 1.3, P=0.386$ ), but the $\mathrm{T} / \mathrm{N}$ ratio was significantly greater in the $\mathrm{PR}+$ cancers $(2.6 \pm 0.9$ vs. $1.5 \pm 0.3, P=$ 0.001). In addition, there was a significant correlation between distribution volume ratio and $\mathrm{T} / \mathrm{N}$ ratio $(r=0.89 ; P=0.001)$ but not between distribution volume ratio and either PR status or standardized uptake value, likely because of small sample size. On the basis of whole-body PET data in 12 patients, the gallbladder appeared to be the dose-limiting organ, with an average radiation dose of $0.113 \mathrm{mGy} / \mathrm{MBq}$. The whole-body dose was 0.015 $\mathrm{mGy} / \mathrm{MBq}$, and the effective dose was $0.020 \mathrm{mSv} / \mathrm{MBq}$. No adverse effects of ${ }^{18} \mathrm{~F}$-FFNP were encountered. Conclusion: ${ }^{18} \mathrm{~F}-$ FFNP PET is a safe, noninvasive means for evaluating tumor $P R s$ in vivo in patients with breast cancer. The relatively small absorbed doses to normal organs allow for the safe injection of up to $440 \mathrm{MBq}$ of ${ }^{18} \mathrm{~F}-\mathrm{FFNP}$.

Key Words: PET; progesterone receptor; breast cancer

J Nucl Med 2012; 53:363-370

DOI: 10.2967/jnumed.111.098319

Received Sep. 15, 2011; revision accepted Oct. 25, 2011.

For correspondence or reprints contact: Farrokh Dehdashti, Mallinckrodt Institute of Radiology, 510 S. Kingshighway Blvd., St. Louis, MO 63110.

E-mail: dehdashtif@mir.wustl.edu

Published online Feb. 13, 2012.

COPYRIGHT @ 2012 by the Society of Nuclear Medicine, Inc.
B reast cancer remains the leading cause of cancer mortality among women in Western countries. Distinct characteristics of breast cancer can be exploited to help determine the overall prognosis and the likelihood of response to specific therapies. It is well established that several factors, including the level and activities of steroid receptors, peptide growth factors, oncogenes, and tumor suppressor genes, play a crucial role in determining tumor response to various endocrine therapies and the development of resistance to these treatments in breast cancer (1).

In general, hormone-sensitive breast cancer is less aggressive than hormone-resistant disease; hormone-sensitive disease occurs more commonly in postmenopausal women and is characterized by longer disease-free and overall survival (2-5). Even with metastatic disease, the median survival in patients with estrogen receptor-positive $(\mathrm{ER}+)$ tumors is 3 times longer than that in patients with estrogen receptornegative (ER-) breast cancer ( 6 ). In treating metastatic breast cancer, the hormone receptor status directs systemic therapy. $\mathrm{ER}+$ disease will respond to first-line endocrine therapy in $55 \%-60 \%$ of patients (7). The presence of the progesterone receptor (PR) increases the likelihood of hormone responsiveness to approximately $75 \%$ (6). Progesterone receptornegative $(\mathrm{PR}-)$ tumors are less responsive to therapy, perhaps suggesting that PR may be necessary for positive therapeutic outcome with endocrine therapy. Alternatively, because ER is a key transcription factor for the activation of PR, lack of $\mathrm{PR}$ expression in ER+ cells also could suggest that the estrogen response pathway may not be functional in these tumors. Only a small fraction of tumors are ER- and progesterone receptor-positive $(\mathrm{PR}+)$, and they demonstrate an intermediate response to endocrine therapy (6).

The PR concentration in tumor tissue is determined by in vitro assays. In recent years, immunohistochemical receptor assays have increasingly replaced quantitative radioligand binding assays $(8,9)$. Immunohistochemical assays of ER and PR have several shortcomings, most notably that they provide limited information about the functional status of the 
receptors and the responsiveness of tumor to hormone therapy. In addition, in recurrent or metastatic breast cancer, the ER or PR status of the lesions may not always be the same as that of the original primary tumor. Indeed, the receptor status of recurrent or metastatic disease may be more predictive of response to hormone therapy. However, because metastatic lesions often are not amenable to biopsy and because the biopsy of multiple lesions is impractical, the receptor status of the lesions cannot be easily determined. Therefore, a method that can reliably determine both the quantity and the functional status of tumor ERs and PRs in individual lesions would be of critical importance in identifying patients likely to benefit from hormonal therapy.

We have recently synthesized and characterized the radiofluorinated steroid compound $21{ }^{18}$ F-fluoro- $16 \alpha, 17 \alpha-$ $\left[(R)-\left(1^{\prime}-\alpha\right.\right.$-furylmethylidene)dioxy]-19-norpregn-4-ene-3,20dione $\left({ }^{18} \mathrm{~F}-\mathrm{FFNP}\right)$, which has high affinity and selectivity for PR (10). ${ }^{18} \mathrm{~F}$-FFNP was identified to be the most promising progestin derivative for PET in preclinical studies (11-14). It is designed to be stable against defluorination, with resultant low bone uptake $(11,12)$. Low levels of activity accumulate in the liver and fat, as its relatively low lipophilicity translates into low nonspecific binding in vivo. Thus, we chose ${ }^{18}$ F-FFNP for a first-in-human study to document safety and estimate human dosimetry and to correlate primary tumor ${ }^{18}$ F-FFNP uptake with tissue PR assays.

\section{MATERIALS AND METHODS}

\section{Patient Population}

This study was conducted under an investigational new drug application (IND 76,214) submitted to the U.S. Food and Drug Administration and was approved by the Institutional Review Board and the Radioactive Drug Research Committee at Washington University School of Medicine. The preclinical data in the investigational new drug application are briefly summarized in the supplemental material (supplemental materials are available online only at http://jnm.snmjournals.org). All patients gave written informed consent before participation. We studied 15 postmenopausal and 5 adult premenopausal women with newly diagnosed breast cancer. All patients except 1 had the ER and PR status of their tumors confirmed by qualitative immunohistochemical staining of the primary breast cancer; the immunohistochemical assays were graded using the semiquantitative Allred scoring system (9). In the remaining patient, ER and PR status was determined by assisted quantitative image analysis. Patients were required to have a primary lesion size of $1.5 \mathrm{~cm}$ or greater as determined by imaging studies (mammography, ultrasonography, $\mathrm{CT}$, or MRI) or physical examination.

\section{${ }^{18}$ F-FFNP Synthesis}

${ }^{18} \mathrm{~F}$-FFNP was produced using an adaptation of a published procedure $(12,14)$. The diastereomerically pure 21-mesylate, endo-9a precursor was reacted with non-resin-treated $\left[{ }^{18} \mathrm{~F}\right]$-fluoride, Kryptofix $_{222}$ (Aldrich), and potassium carbonate in acetonitrile at $85^{\circ} \mathrm{C}$ for $5 \mathrm{~min}$. The reaction mixture was prepurified by passing through a silica light SepPak (Waters), followed by reversed-phase high-performance liquid chromatography purification. ${ }^{18} \mathrm{~F}-\mathrm{FFNP}$ was extracted from the high-performance liquid chromatography mobile phase using solid-phase extraction and was reconstituted in $10 \%$ ethanol in saline. Starting from $11.1 \mathrm{GBq}(300 \mathrm{mCi})$ of ${ }^{18} \mathrm{~F}-$ fluoride and using $0.4 \mathrm{mg}$ of potassium carbonate and prompt work-up to avoid the decomposition of the acid- and base-labile ${ }^{18} \mathrm{~F}-\mathrm{FFNP}$, we produced $0.74-1.11 \mathrm{GBq}(20-30 \mathrm{mCi})$ of ${ }^{18} \mathrm{~F}-\mathrm{FFNP}$ at the end of synthesis (90 min). The final formulation of FFNP is stabilized in ethanol or saline. Non-resin-treated ${ }^{18} \mathrm{~F}$-fluoride was used to achieve high specific activity. The specific activity was measured by high-performance liquid chromatography to be 185$740 \mathrm{GBq}(5,000-20,000 \mathrm{mCi}) / \mu \mathrm{mol}$, and the effective specific activity was measured by receptor binding assay to be up to $740 \mathrm{GBq}$ $(20,000 \mathrm{mCi}) / \mu \mathrm{mol}$.

\section{PET Procedure}

For safety evaluation, all patients underwent vital sign measurement (blood pressure, heart and respiratory rate, and temperature), clinical laboratory testing (standard hematologic and comprehensive metabolic panels that included hemoglobin, white blood cells, neutrophils, lymphocytes, platelets, creatinine, blood urea nitrogen, calcium, sodium, potassium, carbon dioxide, alanine transaminase, aspartate aminotransferase, alkaline phosphatase, total bilirubin, and albumin), urinalysis, and electrocardiography before ${ }^{18}$ F-FFNP administration, as well as during and after completion of imaging.

${ }^{18}$ F-FFNP PET was performed before the initiation of therapy. PET was performed with an EXACT HR + scanner (CTI/Siemens). Performance specifications have been reported $(15,16)$. Each patient received ${ }^{18} \mathrm{~F}$-FFNP intravenously over a period of at least $60 \mathrm{~s}$, via an injection site in the arm opposite that of the known breast lesion.

Twelve patients underwent whole-body PET at 2 separate times after ${ }^{18} \mathrm{~F}$-FFNP injection for human dosimetry calculation. Patients were imaged at 0 and 120, 30 and 150, 60 and 180, or 90 and $210 \mathrm{~min}$ after injection. Whole-body imaging consisted of transmission (2-4 min per bed position) and emission (5-8 min per bed position) imaging. Emission images started at the level of the pelvis and proceeded to the top of the brain and were followed by transmission imaging. Imaging time depended on patient size, injected dose, and time after injection.

The remaining 8 patients underwent dynamic ${ }^{18} \mathrm{~F}$-FFNP PET acquisition centered at the level of the known primary breast lesions for $60 \min (30 \times 2,12 \times 10,6 \times 20,10 \times 60$, and $9 \times$ $300 \mathrm{~s}$ frames). Time-activity curves were generated from the dynamic data to determine the optimal time for static imaging.

The emission images were corrected for attenuation using segmentation. PET images were reconstructed using an orderedsubset estimation maximization iterative algorithm. Images were smoothed with a 5-mm post-reconstruction filter.

\section{Image Analysis}

PET images were evaluated qualitatively with the following grading scale: no uptake (tumor $\leq$ background), minimal uptake (tumor $=$ background), moderate uptake (tumor $>$ background), and intense uptake (tumor $>>$ background). Tumor uptake was dichotomized as increased (moderate or intense uptake) or absent (no uptake or minimal uptake). The images were evaluated semiquantitatively by measurement of the tumor maximum standardized uptake value $\left(\mathrm{SUV}_{\max }\right)$ and the tumor-to-normal-breast $(\mathrm{T} / \mathrm{N})$ activity ratio. A region of interest (ROI) was drawn around the entire lesion, with knowledge of the primary tumor location. $\mathrm{SUV}_{\text {max }}$ was determined within the ROI. A similar ROI was drawn 
in the normal contralateral breast. The $\mathrm{T} / \mathrm{N}$ ratio was calculated by dividing the $\mathrm{SUV}_{\max }$ of the primary tumor by the average standardized uptake value (SUV) of the normal breast. Finally, quantitative Logan graphical analysis was performed with a reference ROI in the normal breast (17). After some time (last 45-60 min of image acquisition), a linear-regression model was optimized against the normalized tumor and reference region time-activity curves (17). In practice, the term that includes the population clearance average was omitted (18). The slope of the linear-regression line provided the distribution volume ratio (DVR) between target tumor and reference regions.

\section{Image-Derived Patient Dosimetry}

Organ activity concentration data were obtained by drawing ROIs on the PET images for the following organs: liver, gallbladder, bone or bone marrow, small intestines, uterus, and urinary bladder. No other organs were visible on the images. The ROIs on the liver, bone or bone marrow, uterus, and small intestines were drawn over several adjacent slices to encompass most of the visible part of the organs. For these organs, total activity accumulated in an organ was calculated by multiplying the average activity concentration by the organ mass for a standard 56-kg adult woman and scaling by the mass of the patient relative to the standard model. The total activity in each organ was divided by the injected activity to derive the percentage injected dose. For the gallbladder and urinary bladder, the total activity was measured using a large ROI encompassing the whole organ. Time-activity curves were then obtained combining the data from all 12 patients by applying decay correction to the injection time. Finally, organ residence times were obtained by analytic integration of a clearance or uptake function fitted as a combination of exponential functions. All unmeasured activity was assigned to the remainder of the body. The measured residence times were used in OLINDA/EXM with the standard adult female model to yield the human radiation dose estimates.

\section{Statistical Analysis}

To determine the relationship between ${ }^{18} \mathrm{~F}$-FFNP PET and in vitro PR status, means and SD of ${ }^{18} \mathrm{~F}$-FFNP tumor uptake measures $\left(\mathrm{SUV}_{\max }\right.$ and $\left.\mathrm{T} / \mathrm{N}\right)$ were calculated for $\mathrm{PR}+$ and $\mathrm{PR}-$ patients, respectively, and the group means were compared using 2-sample $t$ tests. The association between qualitative ${ }^{18} \mathrm{~F}$-FFNP tumor uptake and PR status was assessed using the Kruskal-Wallis rank-sum test. The association between ${ }^{18} \mathrm{~F}$-FFNP PET results (qualitative analysis, $\mathrm{SUV}_{\max }$, and $\mathrm{T} / \mathrm{N}$ ) and PR expression (semiquantitative Allred score) was assessed using Spearman rank correlation coefficients. The association of DVR versus PR expression, $\mathrm{SUV}_{\max }$, and T/N was also described by Spearman correlation. Because of the small sample size, the $P$ values for all the above analyses were derived using a permutation analysis. Random samples $(10,000)$ were generated by reshuffling the PR status, and the permuted $P$ value was the fraction of permuted samples that resulted in a $P$ value smaller than that of the original sample (19).

\section{RESULTS}

\section{Patient Characteristics}

Twenty-one patients were enrolled in the study. One patient was excluded; she consented but then did not participate. None of the patients had received any hormonal therapy before participating in this trial. The demographic characteristics of the 20 evaluable patients are summarized in Table 1. Their median age was 55 y (age range, 32-71 y). There were

TABLE 1

Patient Demographic Data

\begin{tabular}{|c|c|c|c|c|c|c|c|}
\hline Patient & Age $(y)$ & Injected dose (MBq) & Mass $(\mu g)$ & $\mathrm{ER} / \mathrm{PR} / \mathrm{HER} 2$ & Tumor T/N & Tumor SUV & DVR \\
\hline \multicolumn{8}{|c|}{$\begin{array}{l}\text { Dynamic } \\
\text { patients }\end{array}$} \\
\hline 1 & 59 & 359 & 5.38 & $-1-1-1$ & 1.9 & 3.4 & 2.4 \\
\hline 2 & 39 & 281 & 0.84 & $+/+1-1,+1+1-/^{*}$ & $2.3,2.5^{\star}$ & $3.0,3.2^{\star}$ & $1.9,2.7$ \\
\hline 3 & 59 & 222 & 3.41 & $-1-1-1$ & 1.9 & 1.5 & 1.4 \\
\hline 4 & 32 & 178 & 0.83 & $+1-1-1$ & 1.1 & 3.9 & 1.4 \\
\hline 5 & 57 & 366 & 0.53 & $+/+/-1,+/+/-/^{*}$ & $3.5,2.8^{\star}$ & $3.4,2.7^{\star}$ & $3.7,2.8$ \\
\hline 6 & 58 & 148 & 1.91 & $+1+1-1$ & 1.2 & 2.1 & 1.1 \\
\hline 7 & 71 & 366 & 0.56 & $+1+1-1$ & 2.2 & 2.9 & 2.0 \\
\hline 8 & 58 & 370 & 0.34 & $+1+1-1$ & 1.7 & 1.2 & 1.6 \\
\hline \multicolumn{8}{|c|}{$\begin{array}{l}\text { Dosimetry } \\
\text { patients }\end{array}$} \\
\hline 1 & 49 & 89 & 1.39 & $+1+1-1$ & 2.4 & 1.2 & Not applicable \\
\hline 2 & 52 & 185 & 1.87 & $+1+1-1$ & 1.9 & 2.3 & Not applicable \\
\hline 3 & 64 & 370 & 2.67 & $+1+1-1$ & 1.8 & 2.4 & Not applicable \\
\hline 4 & 49 & 104 & 0.83 & $-1-/-1$ & 1.5 & 1.6 & Not applicable \\
\hline 5 & 53 & 329 & 0.75 & $-1-1-1$ & 1.5 & 1.2 & Not applicable \\
\hline 6 & 47 & 270 & 1.32 & $-1-1-1$ & 1.2 & 0.5 & Not applicable \\
\hline 7 & 68 & 359 & 0.90 & $+1+1-1$ & 1.5 & 1.2 & Not applicable \\
\hline 8 & 64 & 370 & 0.31 & $+1+1-1$ & 4.0 & 4.4 & Not applicable \\
\hline 9 & 44 & 215 & 0.90 & $+1+1-1$ & 2.6 & 1.8 & Not applicable \\
\hline 10 & 62 & 352 & 0.54 & $+/+/-/$ & 4.0 & 3.2 & Not applicable \\
\hline 11 & 55 & 315 & 0.76 & $+1+1-1$ & 3.2 & 1.9 & Not applicable \\
\hline 12 & 49 & 259 & 0.70 & $+/+/-1$ & 3.8 & 2.3 & Not applicable \\
\hline
\end{tabular}

*Second breast cancer in patients with 2 breast cancers. 

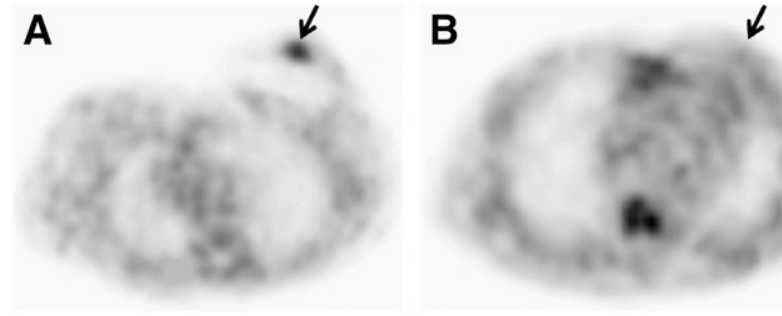

FIGURE 1. Representative transverse ${ }^{18} \mathrm{~F}$-FFNP PET images in patient with $\mathrm{PR}+$ breast cancer $(\mathrm{A})$ and another with PR- breast cancer (B). Arrows point to tumor.

22 primary breast cancers in total; 2 patients each had 2 cancers in different quadrants of the same breast. The tumors were $\mathrm{ER}+$ and $\mathrm{PR}+$ in 16 and $\mathrm{ER}-$ and $\mathrm{PR}-$ in 6.

\section{Safety Evaluation}

The mean and SD of the administered mass of ${ }^{18} \mathrm{~F}-\mathrm{FFNP}$ was $1.34 \pm 1.24 \mu \mathrm{g}$ (range, 0.34-5.38 $\mu \mathrm{g}$ ). The mean administered activity was $275.3 \pm 95.2 \mathrm{MBq}$ (range, 89$370 \mathrm{MBq}$ ). There were no adverse or clinically detectable pharmacologic effects in any of the 20 patients. No significant changes in vital signs or the results of laboratory studies or electrocardiograms were observed.

\section{PET Results}

The 22 breast masses ranged from 1.5 to $11.6 \mathrm{~cm}$ in maximum diameter (mean $\pm \mathrm{SD}, 3.1 \pm 2.1 \mathrm{~cm}$ ). The ${ }^{18} \mathrm{~F}$-FFNP uptake of each breast mass was compared with that of the corresponding site in the normal contralateral breast. Uptake in the breast mass was moderately increased relative to normal breast tissue in 9 and markedly increased in 7 , whereas no uptake was seen in the remaining 6 tumors. Fifteen ER + and PR + and 1 ER- and PR- tumors had moderately or markedly increased ${ }^{18} \mathrm{~F}$-FFNP uptake, whereas no or minimal uptake was seen in $1 \mathrm{ER}+$ and $\mathrm{PR}+$ and 5 ER- and PR- tumors (Fig. 1). Tumor ${ }^{18}$ F-FFNP uptake assessed qualitatively was significantly different in patients with $\mathrm{PR}+$ and $\mathrm{PR}-$ cancers $(P=0.001)$.

Tumor ${ }^{18}$ F-FFNP uptake assessed by $\mathrm{SUV}_{\max }$ was not significantly different in patients with $\mathrm{PR}+$ and PR- cancers (mean $\pm \mathrm{SD}, 2.5 \pm 0.9$ vs. $2.0 \pm 1.3, P=0.386$ ) (Fig. 2 ). However, the $\mathrm{T} / \mathrm{N}$ ratio was significantly different in patients with $\mathrm{PR}+$ and $\mathrm{PR}-$ cancers $(2.6 \pm 0.9$ vs. $1.5 \pm$ $0.3, P=0.001)$. In addition, there was a significant correlation between semiquantitative Allred scoring and the T/N ratio but not with $\mathrm{SUV}_{\text {max }}(P=0.027$ and 0.235 , respectively). A significant correlation was seen between DVR and T/N ( $r=0.89 ; P=0.001)$ but not between DVR and either PR expression $(r=0.34 ; P=0.18)$ or SUV ( $r=0.38 ; P=0.13$ ). Although the T/N correlated with DVR measures, DVR for ${ }^{18}$ F-FFNP was not significantly different between PR- and PR + tumors, likely because of the small number of subjects, in particular with $\mathrm{PR}-$ tumors.

Analysis of dynamic imaging $(n=8)$ indicated that peak tumor uptake was achieved a few minutes after injection and stayed unchanged throughout the $60 \mathrm{~min}$. Similarly, activity in the blood (measured in an ROI over the left ventricular chamber) showed rapid clearance to approximately $10 \mathrm{kBq} / \mathrm{mL}$ for $370 \mathrm{MBq}$ injected by approximately $5 \mathrm{~min}$ and slowly decreased thereafter over several hours after injection (Supplemental Fig. 1G). Time-activity curves for the tumor, contralateral breast, blood pool, and liver for 1 patient with increased ${ }^{18} \mathrm{~F}-\mathrm{FFNP}$ uptake in an $\mathrm{ER}+$ and $\mathrm{PR}+$ tumor are shown in Figure 3.

\section{Dosimetry Results}

Typical whole-body images (Fig. 4) show dominant activity in the liver, gallbladder, and small intestines. A small amount of activity was observed in bone. The combined time-activity curves, along with the clearance or uptake fits, are presented in Supplemental Figure 1. The PET images of 7 patients were coregistered to clinically obtained CT scans to guide the delineation of the uterus ROIs (Fig. 5). The remaining 5 patients had prior hysterectomy. The ${ }^{18}$ F-FFNP uptake assessed by average SUV was higher in the 5 premenopausal women (mean, 3.5; range, 2.8-4.4) than in the 2 postmenopausal women (mean, 1.1; range, $1.39-0.77$ ).

The largest residence times are observed in the liver and small intestines, reflecting hepatobiliary elimination of ${ }^{18}$ F-FFNP (Table 2). The total cumulative activity in the urine accounted for $7.45 \mathrm{~min}$. The bladder voiding model of OLINDA/EXM was then used, with a bladder voiding interval of $1 \mathrm{~h}$ for a bladder-content residence time of
FIGURE 2. ${ }^{18} \mathrm{~F}-\mathrm{FFNP}$ uptake assessed by $\mathrm{SUV}_{\max }$ (left) and T/N ratio (right) in $\mathrm{PR}+$ and PR- breast cancers.

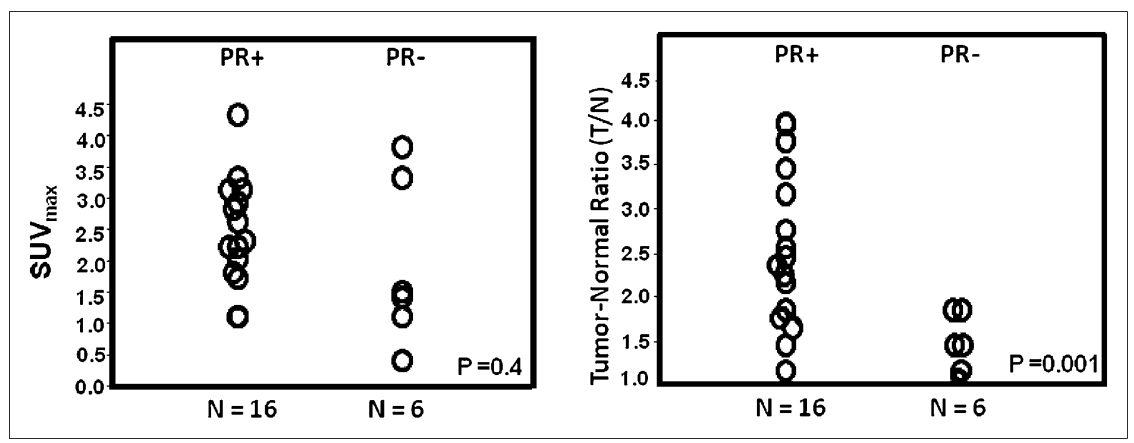




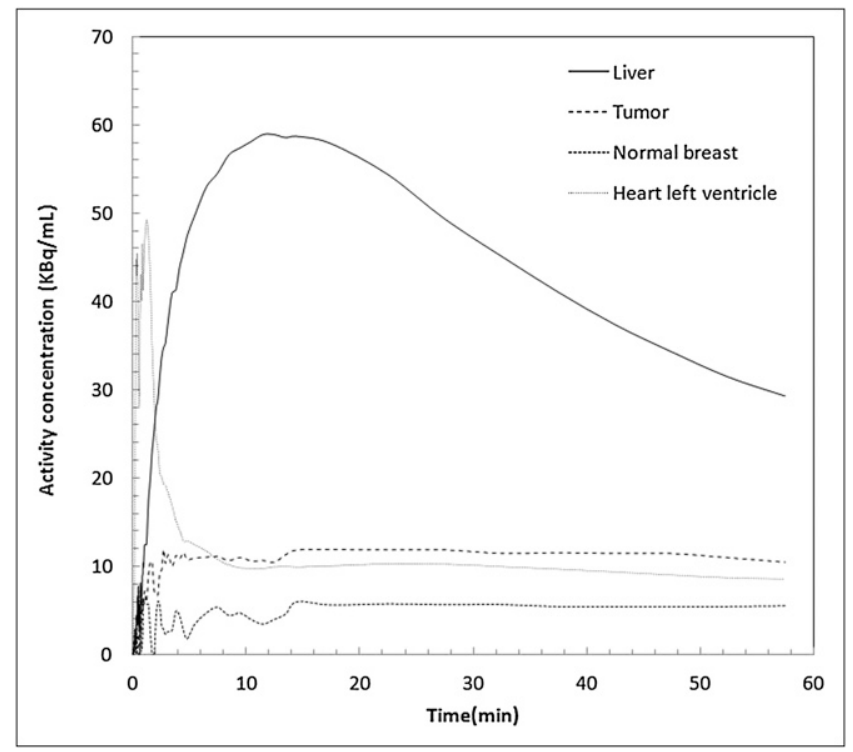

FIGURE 3. Time-activity distribution in liver, tumor, normal breast, and blood (from ventricular chamber). Data were normalized for 370-MBq injection (patient 5, dynamic cohort) with ER+ and PR+ tumor. Uptake of ${ }^{18} \mathrm{~F}-\mathrm{FFNP}$ in this patient's tumor was twice that in her normal breast.

$0.3 \mathrm{~min}$ (and a corresponding excreted cumulative activity of $7.15 \mathrm{~min}$ or approximately $5 \%$ of the injected activity). Overall, $33 \%$ of injected activity was measured in the visible organs. The remaining, unmeasured, activity was associated to the remainder of the body.

On the basis of the human imaging data, the gallbladder is the dose-limiting organ, with an average radiation dose of $0.113 \mathrm{mGy} / \mathrm{MBq}$. The whole-body dose was $0.015 \mathrm{mGy} /$
A

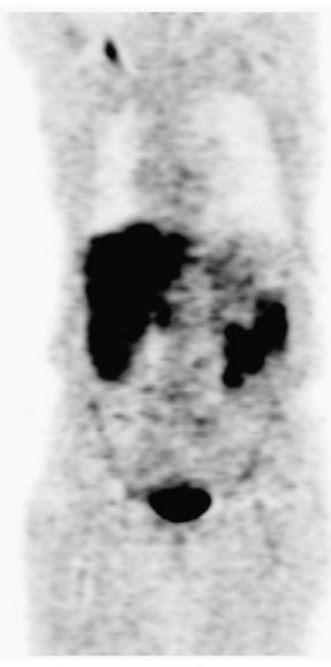

$1 \mathrm{Hr}$
B

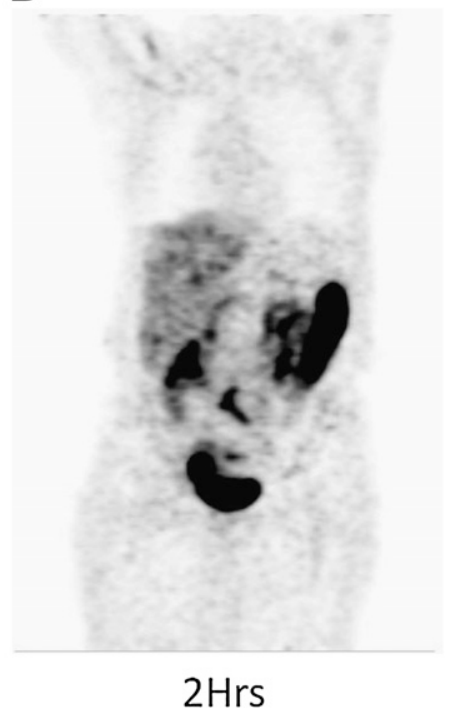

FIGURE 4. Typical whole-body coronal images of patient at $1 \mathrm{~h}(\mathrm{~A})$ and $2 \mathrm{~h}(\mathrm{~B})$ after injection of $370 \mathrm{MBq}(10 \mathrm{mCi})$ of ${ }^{18} \mathrm{~F}-\mathrm{FFNP}$. Accumulation of activity is primarily seen in liver and small intestines

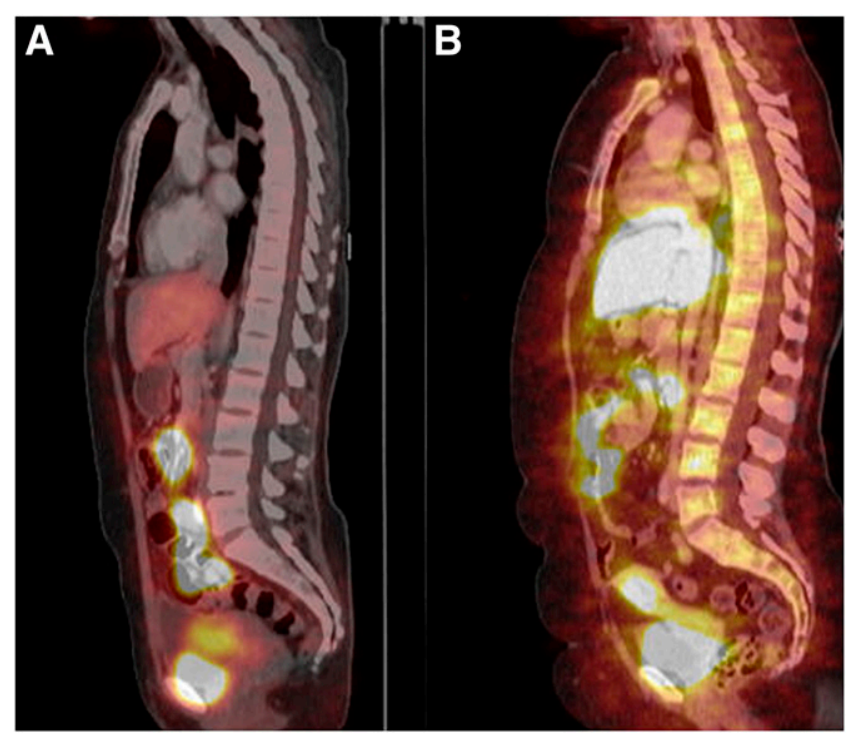

FIGURE 5. Sagittal ${ }^{18} \mathrm{~F}-\mathrm{FFNP}$ images registered to CT images showing accumulation in liver, intestines, uterus, and urinary bladder.

$\mathrm{MBq}$, and the effective dose was $0.020 \mathrm{mSv} / \mathrm{MBq}$. Radiation doses to all organs are listed in Table 3.

\section{DISCUSSION}

Endocrine therapy targeting steroid receptors remains the most effective form of systemic therapy in breast cancer. About $70 \%-80 \%$ of patients with breast cancer have hormone receptor-positive tumors and are candidates for endocrine therapy. ER expression is considered one of the most important biomarkers in breast cancer, both providing prognostic information and predicting responsiveness to endocrine treatment. The expression of PR is strongly dependent on the presence of functional ERs. There is evidence that metastatic breast cancers expressing both ER and PR respond better to antiestrogen therapy than do those that show ER positivity but lack PR expression (20). A strong prognostic value for PR expression also has been reported in adjuvant trials comparing tamoxifen treatment (21).

In the past, ER and PR were most often determined in tumor tissue using ligand binding assays, but standard clinical methods now use immunohistochemistry. The immunohistochemical evaluation criteria to establish the positivity of ER and PR are not uniform in different laboratories, with widely ranging cutoff values for defining positive results (e.g., from $1 \%$ to $10 \%$ positive cells) (22). A recent systematic review of immunohistochemistry for evaluation of ER and PR have found that up to $20 \%$ of current test results worldwide may be inaccurate (false-negatives or false-positives) (9).

On the basis of in vitro analyses of PR and ER, the presence of ER alone predicts for clinical benefit in only $55 \%-60 \%$ of patients; if both ER and PR are present, the likelihood of benefit increases only slightly to $60 \%-70 \%$ (23-25). Moreover, although the choice of therapy and the 
TABLE 2

Organ Residence Times in Minutes from Human Whole-Body PET Images

\begin{tabular}{lc}
\hline \multicolumn{1}{c}{ Organ } & Residence times (min) \\
\hline Liver & $16.3 \pm 5.1$ \\
Small intestine & $20.8 \pm 5.2$ \\
Gallbladder & $4.14 \pm 1.04$ \\
Bone/marrow & $4.60 \pm 1.15$ \\
Uterus & $0.47 \pm 0.12$ \\
Blood (left ventricle) & $11.4 \pm 2.8$ \\
& \\
\hline Data are mean \pm SD. & \\
\hline
\end{tabular}

success in control of metastatic disease rest heavily on the ER and PR status of the metastatic disease, current guidelines do not mandate assessment of ER and PR status of distant metastases. Thus, therapeutic decisions for metastatic breast cancer are most often based on the profile of the primary tumor. An increasing body of literature suggests that tumor characteristics can change over time, especially with regard to ER and PR status (26-31). Because metastatic lesions often are not amenable to biopsy and because the biopsy of multiple lesions is impractical, the receptor status of the lesions cannot be easily determined. Discordance of hormone receptor status between the primary tumor and metastatic disease is not uncommon. A

TABLE 3

Human Organ Radiation Doses Estimated from Human PET Images in mGy/MBq

\begin{tabular}{|lc}
\hline \multicolumn{1}{c}{ Organ } & $\begin{array}{c}\text { Organ dose human } \\
\text { PET }(\mathrm{mGy} / \mathrm{MBq})\end{array}$ \\
\hline Adrenals & 0.017 \\
\hline Brain & 0.010 \\
\hline Breasts & 0.010 \\
\hline Gallbladder & 0.113 \\
\hline Lower large intestine & 0.020 \\
\hline Small intestine & 0.096 \\
\hline Stomach & 0.017 \\
\hline Upper large intestine & 0.030 \\
\hline Heart wall & 0.015 \\
\hline Kidneys & 0.017 \\
\hline Liver & 0.051 \\
\hline Lungs & 0.013 \\
\hline Muscle & 0.013 \\
\hline Ovaries & 0.023 \\
\hline Pancreas & 0.018 \\
\hline Red marrow & 0.015 \\
\hline Osteogenic cells & 0.024 \\
\hline Skin & 0.009 \\
\hline Spleen & 0.014 \\
\hline Thymus & 0.012 \\
\hline Thyroid & 0.011 \\
\hline Urinary bladder wall & 0.016 \\
\hline Uterus & 0.034 \\
\hline Whole body & 0.015 \\
\hline Effective dose (mSv/MBq) & 0.020 \\
\hline
\end{tabular}

recent review found such discordance in the ER status of $2.5 \%-17 \%$ and in the PR status of $5.9 \%-51.7 \%$ (32). Considering the limitations of in vitro methods, a reliable noninvasive method that could assess the receptor status of metastatic lesions before and after therapy would be extremely valuable.

In this study, we found a significant positive correlation between primary breast cancer uptake of ${ }^{18} \mathrm{~F}-\mathrm{FFNP}$, assessed qualitatively and quantitatively using the $\mathrm{T} / \mathrm{N}$ ratio, and the tumor PR status. However, no significant correlation was demonstrated between the $\mathrm{SUV}_{\max }$ and DVR for ${ }^{18} \mathrm{~F}$-FFNP uptake and receptor status. This observation may indicate that the tumor uptake of ${ }^{18}$ F-FFNP needs to be corrected for nonspecific uptake in the contralateral breast or further characterized by compartmental modeling to delineate specific and nonspecific uptake and the contribution (if any) of metabolites to the overall signal.

Future studies to assess the predictive value of ${ }^{18} \mathrm{~F}$-FFNP PET should be designed to correlate ${ }^{18} \mathrm{~F}$-FFNP uptake and response to endocrine therapy. Namer et al. have shown an increase in PR level $1 \mathrm{wk}$ after treatment with tamoxifen, which has an initial weak estrogenic effect, in $40 \%$ of ER+ but in no ER- metastatic lesions in breast cancer patients (33). Waseda et al. noted that longer tamoxifen treatment reduced PR levels, indicating that its agonistic effect is transient (34). Howell et al. showed that $90 \%$ of patients whose tumor PR levels increased after tamoxifen responded to endocrine therapy, versus $35 \%$ in patients whose tumor PR levels declined or were undetectable, regardless of tumor ER status; notably, PR levels after tamoxifen were more predictive than initial PR measurements but less predictive than change in PR with tamoxifen (35). In all of these studies, PR levels were determined by radioligand binding assays, thus, it seems reasonable to consider ${ }^{18} \mathrm{~F}$-FFNP PET as a noninvasive test to predict patient response to endocrine therapies, monitoring either PR levels after an initial hormone treatment or, perhaps, a change in PR levels in an estrogen-challenge test paradigm, as we have done using ${ }^{18} \mathrm{~F}$-FDG PET to monitor changes in tumor metabolism (36-39).

Our dynamic data indicated that peak tumor uptake was achieved early after ${ }^{18}$ F-FFNP injection and stayed unchanged through $60 \mathrm{~min}$. The $\mathrm{T} / \mathrm{N}$ ratio indicated rapid uptake and no significant washout.

The biodistribution data showed the highest activity in the liver, gallbladder, and small intestine. Human radiation doses calculated from the PET images indicated an effective dose of $0.02 \mathrm{mSv} / \mathrm{MBq}$, a value that is comparable to that reported for ${ }^{18} \mathrm{~F}$-fluoroestradiol $(0.022 \mathrm{mSv} / \mathrm{MBq})$ (40). The dose to the liver is approximately $40 \%$ of that reported for ${ }^{18} \mathrm{~F}$-fluoroestradiol, corresponding to a liver residence time $38 \%$ of that reported for ${ }^{18} \mathrm{~F}$-fluoroestradiol. ${ }^{18} \mathrm{~F}$-FFNP and ${ }^{18} \mathrm{~F}$-fluoroestradiol show the highest residence times in the liver, gallbladder, and intestines, suggesting a similar route of elimination. Assessment of uterine activity, in only 3 premenopausal women, showed significant uptake (average SUVs, 2-5). 
The animal biodistribution data and dosimetry estimates, which compare well with the human PET data (albeit with higher values in the large intestines for the animal data), also showed that the highest residence times were in the liver, uterus, and gastrointestinal tract. Because the animal biodistribution studies were performed in rats, the gallbladder distribution cannot be compared. Rather, the rat liver directly clears the activity to the small intestines, resulting in higher residence times and higher dose to the lower gastrointestinal tract. The resulting radiation doses from the animal studies are consequently higher than those from the human experiment (supplemental material).

\section{CONCLUSION}

We have demonstrated that ${ }^{18} \mathrm{~F}$-FFNP is a safe imaging agent for PET, with organ and total-body radiation doses comparable to those from ${ }^{18} \mathrm{~F}-\mathrm{FES}$ and other commonly used clinical radiopharmaceuticals. Although it is unlikely that ${ }^{18}$ F-FFNP PET will have a role in the diagnosis or staging of breast cancer, we have shown that it can be used to assess the PR status of individual breast cancer lesions. Potentially the most important role of ${ }^{18}$ F-FFNP may be in the noninvasive and repetitive evaluation of the PR positivity of individual lesions. ${ }^{18} \mathrm{~F}$-FFNP PET may be a means for determining whether antiestrogen therapy is appropriate before initiation of therapy or after first- or second-line endocrine therapy.

\section{DISCLOSURE STATEMENT}

The costs of publication of this article were defrayed in part by the payment of page charges. Therefore, and solely to indicate this fact, this article is hereby marked "advertisement" in accordance with 18 USC section 1734.

\section{ACKNOWLEDGMENTS}

This work was supported by U.S. Department of Energy grant DE FG02 84ER60218, National Institutes of Health grant CA 025836, and the Alvin J. Siteman Cancer Center Biostatistic Core and Imaging and Response Assessment Core. No other potential conflict of interest relevant to this article was reported.

\section{REFERENCES}

1. Weigel MT, Dowsett M. Current and emerging biomarkers in breast cancer: Prognosis and prediction. Endocr Relat Cancer. 2010;17:R245-R262.

2. Esteva FJ, Hortobagyi GN. Prognostic molecular markers in early breast cancer. Breast Cancer Res. 2004;6:109-118.

3. Payne SJ, Bowen RL, Jones JL, Wells CA. Predictive markers in breast cancerthe present. Histopathology. 2008;52:82-90.

4. Grann VR, Troxel AB, Zojwalla NJ, Jacobson JS, Hershman D, Neugut AI. Hormone receptor status and survival in a population-based cohort of patients with breast carcinoma. Cancer. 2005;103:2241-2251.

5. Early Breast Cancer Trialists' Collaborative Group. Effects of chemotherapy and hormonal therapy for early breast cancer on recurrence and 15-year survival: An overview of the randomised trials. Lancet. 2005;365:1687-1717.

6. Keen JC, Davidson NE. The biology of breast carcinoma. Cancer. 2003;97:825833.
7. Goldhirsch A, Colleoni M, Gelber RD. Endocrine therapy of breast cancer. Ann Oncol. 2002;13(suppl 4):61-68.

8. Rhodes A, Jasani B, Balaton AJ, Miller KD. Immunohistochemical demonstration of oestrogen and progesterone receptors: correlation of standards achieved on in house tumours with that achieved on external quality assessment material in over 150 laboratories from 26 countries. J Clin Pathol. 2000;53:292-301.

9. Hammond ME, Hayes DF, Dowsett M, et al. American Society of Clinical Oncology/College of American Pathologists guideline recommendations for immunohistochemical testing of estrogen and progesterone receptors in breast cancer (unabridged version). Arch Pathol Lab Med. 2010;134:e48-e72.

10. Lee JH, Zhou HB, Dence CS, Carlson KE, Welch MJ, Katzenellenbogen JA. Development of [f-18]fluorine-substituted tanaproget as a progesterone receptor imaging agent for positron emission tomography. Bioconjug Chem. 2010; 21:1096-1104.

11. Kochanny MJ, VanBrocklin HF, Kym PR, et al. Fluorine-18-labeled progestin ketals: synthesis and target tissue uptake selectivity of potential imaging agents for receptor-positive breast tumors. J Med Chem. 1993;36:1120-1127.

12. Buckman BO, Bonasera TA, Kirschbaum KS, Welch MJ, Katzenellenbogen JA. Fluorine-18-labeled progestin 16 alpha, 17 alpha-dioxolanes: development of high-affinity ligands for the progesterone receptor with high in vivo target site selectivity. J Med Chem. 1995;38:328-337.

13. Kym PR, Carlson KE, Katzenellenbogen JA. Progestin 16 alpha, 17 alpha-dioxolane ketals as molecular probes for the progesterone receptor: synthesis, binding affinity, and photochemical evaluation. J Med Chem. 1993;36:1111-1119.

14. Vijaykumar D, Mao W, Kirschbaum KS, Katzenellenbogen JA. An efficient route for the preparation of a 21-fluoro progestin-16 alpha,17 alpha-dioxolane, a high-affinity ligand for pet imaging of the progesterone receptor. J Org Chem. 2002;67:4904-4910.

15. Moreno-Cantú JJ, Thompson CJ, Zatorre RJ. Evaluation of the ECAT EXACT $\mathrm{HR}+3$-D PET scanner in H2(15)O brain activation studies: Dose fractionation strategies for rCBF and signal enhancing protocols. IEEE Trans Med Imaging. 1998;17:979-985.

16. Adam L-E, Zaers J, Ostertag H, Trojan H, Bellemann ME, Brix G. Performance evaluation of the whole-body pet scanner ECAT EXACT HR + following the IEC standard. IEEE Trans Nucl Sci. 1997;44:1172-1179.

17. Logan J. Graphical analysis of pet data applied to reversible and irreversible tracers. Nucl Med Biol. 2000;27:661-670.

18. Logan J. A review of graphical methods for tracer studies and strategies to reduce bias. Nucl Med Biol. 2003;30:833-844.

19. Westfall PH, Young SS. Resampling-Based Multiple Testing: Examples and Methods for p-Value Adjustment. New York, NY: John Wiley \& Sons, Inc.; 1993.

20. Elledge RM, Green S, Pugh R, et al. Estrogen receptor (ER) and progesterone receptor (PGR), by ligand-binding assay compared with ER, PGR and ps2, by immuno-histochemistry in predicting response to tamoxifen in metastatic breast cancer: a southwest oncology group study. Int J Cancer. 2000;89:111-117.

21. Dowsett M, Houghton J, Iden C, et al. Benefit from adjuvant tamoxifen therapy in primary breast cancer patients according oestrogen receptor, progesterone receptor, EGF receptor and HER2 status. Ann Oncol. 2006;17:818-826.

22. Allred DC, Carlson RW, Berry DA, et al. NCCN task force report: estrogen receptor and progesterone receptor testing in breast cancer by immunohistochemistry. J Natl Compr Canc Netw. 2009;7(suppl 6):S1-S21.

23. Vollenweider-Zerargui L, Barrelet L, Wong Y, Lemarchand-Beraud T, Gomez F. The predictive value of estrogen and progesterone receptors' concentrations on the clinical behavior of breast cancer in women: clinical correlation on 547 patients. Cancer. 1986;57:1171-1180.

24. Alanko A, Heinonen E, Scheinin T, Tolppanen EM, Vihko R. Significance of estrogen and progesterone receptors, disease-free interval, and site of first metastasis on survival of breast cancer patients. Cancer. 1985;56:1696-1700.

25. Ravdin PM, Green S, Dorr TM, et al. Prognostic significance of progesterone receptor levels in estrogen receptor-positive patients with metastatic breast cancer treated with tamoxifen: results of a prospective southwest oncology group study. J Clin Oncol. 1992;10:1284-1291.

26. Khasraw M, Brogi E, Seidman AD. The need to examine metastatic tissue at the time of progression of breast cancer: is re-biopsy a necessity or a luxury? Curr Oncol Rep. 2011;13:17-25.

27. Gancberg D, Di Leo A, Cardoso F, et al. Comparison of her-2 status between primary breast cancer and corresponding distant metastatic sites. Ann Oncol. 2002;13:1036-1043.

28. Gong Y, Booser DJ, Sneige N. Comparison of her-2 status determined by fluorescence in situ hybridization in primary and metastatic breast carcinoma. Cancer. 2005;103:1763-1769.

29. Zidan J, Dashkovsky I, Stayerman C, Basher W, Cozacov C, Hadary A. Comparison of her- 2 overexpression in primary breast cancer and metastatic sites and its effect on biological targeting therapy of metastatic disease. $\mathrm{Br} J$ Cancer. 2005;93:552-556. 
30. Amir E, Clemons M. Should a biopsy be recommended to confirm metastatic disease in women with breast cancer? Lancet Oncol. 2009;10:933-935.

31. Broom RJ, Tang PA, Simmons C, et al. Changes in estrogen receptor, progesterone receptor and her- $2 /$ neu status with time: discordance rates between primary and metastatic breast cancer. Anticancer Res. 2009;29:1557-1562.

32. van de Ven S, Smit VT, Dekker TJ, Nortier JW, Kroep JR. Discordances in er, pr and her2 receptors after neoadjuvant chemotherapy in breast cancer. Cancer Treat Rev. 2011;37:422-430.

33. Namer M, Lalanne C, Baulieu EE. Increase of progesterone receptor by tamoxifen as a hormonal challenge test in breast cancer. Cancer Res. 1980;40:17501752 .

34. Waseda N, Kato Y, Imura H, Kurata M. Effects of tamoxifen on estrogen and progesterone receptors in human breast cancer. Cancer Res. 1981;41:1984-1988.

35. Howell A, Harland RN, Barnes DM, et al. Endocrine therapy for advanced carcinoma of the breast: relationship between the effect of tamoxifen upon concentrations of progesterone receptor and subsequent response to treatment. Cancer Res. 1987;47:300-304.
36. Mortimer JE, Dehdashti F, Siegel BA, Trinkaus K, Katzenellenbogen JA, Welch MJ. Metabolic flare: Indicator of hormone responsiveness in advanced breast cancer. J Clin Oncol. 2001;19:2797-2803.

37. Dehdashti F, Mortimer JE, Trinkaus K, et al. Pet-based estradiol challenge as a predictive biomarker of response to endocrine therapy in women with estrogenreceptor-positive breast cancer. Breast Cancer Res Treat. 2009;113:509-517.

38. Dehdashti F, Flanagan FL, Mortimer JE, Katzenellenbogen JA, Welch MJ, Siegel BA. Positron emission tomographic assessment of "metabolic flare" to predict response of metastatic breast cancer to antiestrogen therapy. Eur J Nucl Med. 1999;26:51-56.

39. Ellis MJ, Gao F, Dehdashti F, et al. Lower-dose vs high-dose oral estradiol therapy of hormone receptor-positive, aromatase inhibitor-resistant advanced breast cancer: A phase 2 randomized study. JAMA. 2009;302:774-780.

40. Mankoff DA, Peterson LM, Tewson TJ, et al. [18f]fluoroestradiol radiation dosimetry in human pet studies. J Nucl Med. 2001;42:679-684. 